\title{
Bemerkungen über die Funktion der Schilddrüse und ihrer Nebendrüsen.
}

Bemerkungen zu dem Aufsatze der Herren L. B lumreich und M. Jacoby:

„Experimentelle Untersuchungen ïber die Bedeutung der Schilddrüse und ihrer Nebendrüsen für den Organismus".

Von

\section{Dr. E. Gley,}

Professeur agrégé de physiologie à la Faculté de Médecine de l'Université de Paris.

Wer die Frage der Schilddrüsenfunktion kennt, wird mir ohne Weiteres zustimmen, wenn ich beabsichtige, einiges über die jüngst erschienene Arbeit der beiden Schïler H. Munk's, Bl umr e i ch's und Jacoby's ${ }^{1}$ ) zu sagen. Ich möehte besonders über zwei der hauptsächlichsten Punkte dieser Arbeit sprechen.

1. Der erste betrifft die Thyreoidectomie bei Kaninchen. Fast alle von den Autoren operirten Thiere starben. Die Uebereinstimmung ist vollkommen zwischen diesem allgemeinen Resultat und denjenigen Resultaten, die ich schon vor 5 Jahren als die Folge der Schilddrüsenexstirpation beim Kaninchen bestimmt babe. Ich konstatire dies um so lieber, als die Beobachter gerade anf dem scheinbaren Unterschied zwischen ihren und meinen Experimenten zu verweilen scheinen.

Indessen ist unter den Folgen der Thyreoidectomie eine, von den Beobachtern nicht konstatirte, nämlich die Tetanie. Was mich anbetrifft, so habe ich dieselbe zu oft beobachtet, um sie nicht, trotz B. und J., als eine der gewöhnlichen Folgen der Operation aufzufassen. Die von mir veröffentlichten Versuchsprotokolle haben den Entwicklungsgang dieses Pliänomens klar bewiesen,

1) E. Pflüger's Archiv, Bd, 64. 1896. 
Bemerkungen über die Funktion der Schilddrüse u. ihrer Nebendrüsen. 309

und die in meinem ersten Aufsatze 1) beigegebenen Curven sind in dieser Hinsicht beweiskräftig genug. Seitdem habe ich diese Untersuchungen niemals verlassen; hier ist ein neues typisches Beispiel.

\section{Versuch.}

18. Juni 1894. 4 Kaninchen:

Nr. 1. Gewicht $2180 \mathrm{gr}$, von $2 \mathrm{~h} 45 \mathrm{~m}$ bis $3 \mathrm{~h} 10$ operirt.

Nr. 2. Gewicht $2120 \mathrm{gr}$, von $3 \mathrm{~h} 20 \mathrm{~m}$ bis $3 \mathrm{~h} 35$ operirt.

Nr. 3. Gewicht $1950 \mathrm{gr}$, von $3 \mathrm{~h} 40 \mathrm{~m}$ bis $3 \mathrm{~h} 55$ operirt.

Nr. 4. Gewicht $1860 \mathrm{gr}$, von $4 \mathrm{~h} 5 \mathrm{~m}$ bis $4 \mathrm{~h} 20$ operirt.

Complete Exstirpation, ohne Narkose. Die Arteria thyreoidea ist allein unterbunden; die Venen sind gequetscht oder durchtrennt und die Blutung - ist sehr gering. Die Wunde durch fortlaufende Naht mit Seide geschlossen und mit Vaselin mit 15 Procent Salol bedeckt.

Nr. 1. - 19. Juni, Abends $8 \mathrm{~h}$. Es ist in einem schweren Anfall gefunden; es herrschen dabei allgemeine klonische Convulsionen vor.

20. Juni, Morgens. Zuckungen des ganzen Körpers; allgemeine Parese. Dieser Zustand dauert bis zu dem Tode um $1 \mathrm{~h}$ Nachmittags.

Nr. 2. - 20. Juni, Morgens. Lähmung der Vorderpfoten; Zuckungen des ganzen Körpers.

21. Juni. Dyspnoë; allgemeine Paralyse; Abends Tod.

Nr. 3. - 20. Juni, Nachmittags. Zuckungen des ganzen Körpers.

21. Juni. Häufige Polypnoë; allgemeine Zuckungen. Um $2 \frac{1}{2}$ h Tod.

Nr. 4. - 20. Juni, Morgens. Ganz geschwächt. Dyspnoë. Nachmittags noch mehr geschwächt; es ist um $81 / 2 \mathrm{~h}$ Abends schon starr, todt gefunden.

Wenn dieses Phänomen nicht von dem Verlust der Drüse herribbrte, wo sollte es dann herkommen? Es ist sicherlich zuweilen schwer bei der Operation nicht auch den Nervus recurrens zu verletzen. Das ist mir auch passirt. Aber mir scheint es nicht, dass die Verletzung oder das Unterbinden des einen oder beider Nervi recurrentes, Krämpfe und Zuckungen, wie ich sie beobachtet habe, hervorrufen muss. Ich habe Kaninchen beobachtet, die in Folge des Unterbindens eines dieser Nerven schnaubten, und welche nach Exstirpation der Thyreoidea allein, ohne dass die Nebenschilddrüsen abgetragen worden wären, lange keine Störungen zeigten. Hier ein Beispiel davon.

1) Arch. de physiol, 5e série, IV, 1 janvier 1892. 


\section{Vers u ch.}

17. April 1891. Kaninchen mittleren Alters. $2400 \mathrm{gr} . \quad T=39,95$. Thyreoidectomie ohne Narkose. Ausschneiden der rechten Glandula. Nach der Operation Schna uben. Der rechte Nervus recurrens wurde verlezt.

10. April. $2450 \mathrm{gr} . \quad T=39,5$.

20. April. $2440 \mathrm{gr}$.

21. April. $2440 \mathrm{gr}$.

22. April. $2480 \mathrm{gr}$. $T=39,05$.

23. April. $2480 \mathrm{gr} . \quad T=39,25$.

26. April. $2580 \mathrm{gr} . \quad I=39,5$. Die Wunde heilt zu.

Während der ganzen Zeit Schnauben.

13. Mai. $2650 \mathrm{gr}$. Exstirpation der linken Nebendrüse. Gewicht derselben : $0,01 \mathrm{gr}$.

14. Mai. $2660 \mathrm{gr}$.

19. Mai. $2650 \mathrm{gr} . \quad T=39,25$. Um $2 \mathrm{~h}$ heftige Contraction der Kaumuskeln; tagsüber heftiges, zeitweises Zittern der Kaumuskeln; von Zeit zu Zeit heftiges Zucken des ganzen Kopfes.

Vom 25.-30. Mai nichts zu notiren.

2. Juni. Am Morgen todt aufgefunden.

Ich bemerke übrigens, dass das Thier, bei welchem die Verfasser Zuckungen und Dispnoë beobachtet hatten, totale Thyreoidectomie erlitten hatte. Ich bemerke auch, dass an den fünf Thieren, die sie so schnell sterben sahen (1-5 Tage) dieselbe Operation vollzogen worden war. Es ist wahrscheinlich, sagen die Beobachter, dass diese Thiere an den Folgen der Narkose starben. Das ist aber nur eine Vermuthung. Und hier möchte ich darauf hinweisen, dass meine Operationen an nicht narkotisirten Thieren gemacht worden sind. Ich hatte bald bemerkt, dass die Narkose wegen der Einfachheit der Operation und der Schnelligkeit, mit der sie bei einiger Uebung gemacht werden kann, nutzlos ist.

Endlich haben seit meinen Versuchen mehrere Autoren gleichfalls die Tetanie beobachtet. B. und J. wollen den wenig zahlreichen Versuchen F. de Quervai n's ${ }^{1}$ ) und Hofmeiste $r^{\prime}{ }^{2}$ ) keine

1) Fritz de Quervain: Ueber die Veränderungen des Centralnervensystems bei experimenteller Kachexia thyreopriva der Thiere (Inaug. - D is sertat., Bern, 1893 und Archiv f. path. Anat. u. Physiol. Bd. CXXXIII, 1893).

2) F. Hofmeister: Exper. Unters. über die Folgen des Schilddrüsenverlustes (Beitr. zur klịn. Chir., Bd. XI, 1894). 
Bemerkungen über die Funktion der Schilddrüse u. ihrer Nebendrüsen. 311

Bedeutung zuerkennen. Aber dann vergessen oder ignoriren sie die Experimente von C. Verstraeten und O. Vanderlinden $(\text { Gent })^{1}$ ), die von Cadé-ac und $\mathrm{Guinard}\left(\right.$ Lyon) ${ }^{2}$ ), die von A. Rouxeau (Nantes) ${ }^{3}$ ), die ron Walter Edmunds (London) ${ }^{4}$ ), die von F. Capobianco (Napoli) $)^{5}$.

Verstraeten und Vanderlinden schreiben: „L'extirpation des glandes normales seules n'est pas suivie d'accidents, non plus que l'ablation isolée des glandules. La thyroïdectomie totale, au contraire, est fatale à bref délai, et nous pouvons confirmer d'une façon générale les résultats obtenus par Gley. Les lapins opérés de cette façon sont morts, sans exception, après un temps variable, et l'autopsie faite avec soin n'a montré qu'un reste de tissu thyrödien dans tous ces cas. Les lapins supportent très bien l'opération faite aseptiquement: ils peuvent jouir au début d'une santé en apparence parfaite, mais ils meurent après un temps plus ou moins long. Quelques - uns succombent aux accidents aigus, convulsifs, au bout de quelques heures: e'ètait le petit nombre de nos opérés. D'autres présentent une cachexie spéciale avee troubles trophiques, épaississement de la peau,! chute des poils. D'autres résistent, mais l'amaigrissement arrive après un terme plus on moins long et la température baisse parallèlement avec le poids jusqu'à la mort; cette régle souffre peu ou point d'exceptions; l'issue est fatale; elle est précédée d'inappétence et d'une forte perte du poids

1) C. Verstraten and 0. Vanderlinden: Etude sur les fonctions du corps thyrộde (Mémoires de l'Acad. roy. de meéd, de Belgique, XIII, 7e fascicule, 1894).

2) C. Cadéac and L. Guinard: Quelques faits relatifs aux accidents de la thyrödectomie (Comptes rendus de la Société de Biologie, 2 juin 1894, p. 468).

3) A. Rouxeau: Note sur 65 opérations de thyrö̈dectomie chez le lapin (Comptes rendus de la Société de Biologie, 27 juillet 1895, p. 638).

4) Walter Edmunds: Experiments on the thyroid and parathyroid glands (Procedings of the physiological Society, 18 may 1895); Observations and experiments on the pathology of Graves's disease (The Journ. of pathol. and bacteriol., janvier 1896, p. 488).

5) F. Capobianco: La tiroidectomia nei mammiferi (La Ri forma medica, 25 avril 1895, p. 254). 
du corps. Cet état de dénutrition progressive s'accompagne de phénomènes cérébraux, de somnolence, de paresse, de perte de linstinet de conservation et de la défense. Il se termine par le coma et la mort.

"L'extirpation des glandes thyroïdes proprement dites n'est pas suivie d'accidents; pour les provoquer, il faut en plus l'enlèvement des glandules de Gley. Quel est done le rôle de ces glandules?.. Supposons un instant que les glandules soient capables de compenser la perte de la glande chez le lapin adulte: dans ce cas, il est probable que l'on y trouvera des modifications en rapport avec lear nouvelle fonction. Dans le but d'élucider cette question, nous avons enlevé les glandes principales, et après des intervalles de buit jours, de quinze jours, de trois semaines et plus, nous avons extirpé l'une des glandules.

„Après huit jours, nous avons trouvé la glandule gorgée de sang. Les capillaires et les vaisseaux sont distendus par du sang d'un rouge rutilant très vif (figure 3). Cette congestion active s'accentue encore les jours suivants: telle est l'abondance du sang que l'organe paraît quasi en érection. Il s'affaisse aussitôt après l'extirpation, si l'on n'a pas soin de lier au préalable ses vaisseaux. $\mathrm{Au}$ bout d'un temps plus long, la glandule a augmenté considérablement de volume; elle s'est hypertrophiée et est devenue plus consistante. Après deux mois, elle a augmenté du double dans tous les sens; sa vascularité est plus riche encore, plus abondante, et tous les vaisseaux sont gorgés de sang. La coque conjonctive qui embrasse toute la glandule et qui sépare entre eux les lobules participe avant tout à l'hypertrophie générale; les travées de tissu conjonctif ont triplé de puissance (figure 4). L'élargissement est surtout évident autour des gros vaisseaux centraux. Les cordons cellulaires sont épaissis, non par multiplication des cellules, que nous n'avons vue nulle part, mais par hypertrophie vraie de ees dernières. La disposition des cellules paraît également plus régulière. An bout de deux mois, l'organe tout entier a done subi des modifications profondes, tant macroscopiques que microscopiques: d'embryonnaire qu'il était, il est devenu adulte; ses cellules sont mieux formées, plus développées; elles semblent prêtes à remplir la fonction nouvelle qui leur est échue.

,Malgré un examen attentif, nous n'avons trouvé dans nos préparations aucune trace de substance colloüde, soit extra- ou 
intra-cellulaire. Parmi tous les tissus et organes du corps, l'hypophyse non exceptée, les glandules de Gle y seules subissent cette hypertrophie vraie: une organisation plis parfaite, une nutrition plus active, telle est la réaction qu'elles présentent en face de la thyroildectomie. Le fait est important et sa signification ne saurait échapper à quiconque est familiarisé avec les choses de la pathologie générale. Non seulement nous attribuons, malgré les dénégations de quelques anteurs, un rôle très actif de compensation aux glandules de Gley dans tous les cas d'absence on d'incapacité fonctionnelle du corps thyroüde proprement dit, mais nous les considèrons comme partie intégrante de l'organe thyroïdien."

Cadéac und Guinard sagen nar: "Contrairement à M. Mous s u et conformément à ce qu'a vu M. Gley, nous avons constaté que la thyroïdectomie complète est aussi dangereuse pour les lapins que pour les chiens". Aber ich habe den Assistenten des Professors A $r$ o ing, Herrn $G$ u in ard, um ausführlichere Auskünfte gebeten, und jüngst hat mir dieser Herr geschrieben: „Dans les expériences que nous avons faites avec M. Cad é a c, les lapins n'ont pas pris la plus grande part et dans les notes de laboratoire que j'ai conservées je ne trouve que 9 animaux de cette espèce, soumis à la thyroìdectomie.

„Ayant pour but d'étudier particuliẻrement les résultats de l'ablation complète, j'ai toujours enlevé glande et glandules, suivant les indications que vous donnez dans un de vos mémoires; nos animaux sont morts dans le délai moyen de 2 à 3 jours au plus, un seul est inort le lendemain de l'opération.

„Au début des accidents nous avons presque toujours vu la perte générale des forces avec des mouvements partieuliers d'oscillation de la tête et l'byperthermie. Nos animaux ont présenté ensuite des tremblements, isolés d'abord dans certains muscles de la face ou des membres, mais se généralisant ensuite à d'autres régions; ces tremblements, assez énergiques parfois, étaient entreconpés de véritables erises convulsives qui, sur cinq de nos animaux, ont été trés violentes. L'byperthermie s'est toujours accusée et nos sujets mouraient après avoir présenté de l'incoordination motrice et de la paralysie." (Lyon, le 12 novembre 1896).

Was Rouxeau betrifft, hat er 58 Kaninchen operirt und schreibt er: „Sur 18 opérés de thyroïdectomie complète on com- 
plétée, 47, au moins, présentèrent à des degrés divers ce curienx ensemble de phénomènes nerveux si bien déerits par M. Gle y. Je dis au moins, parce que 2 des opérés moururent dans la nuit qui suivit l'opération et ne purent être observés. J'ajoute que parmi ceux qui guérirent (11), 2 seulement furent indemnes et ne présentérent aucun symptôme spécifique . . L L'ensemble symptomatique a presque toujours débuté au bout de 16 heures; très rarement il a fallu attendre 24 heures et exceptionnellement 40 heures. Les rémissions temporaires parfois observées dans les symptômes n'ont jamais été ni bien accentuées ni de bien longue durée... Les symptômes ont été, d'une façon générale, d'abord des phénoménes convulsifs débutant d'ordinaire par une trémulation marquée des poils dn museau, et un certain degré d'atrésie de l'orifice palpébral, puis des phénoménes paralytiques quelquefois très précoces et débutant souvent par les extenseurs, de l'hypothermie, de la salivation, de la dilatation pupillaire avec vascularisation plus ou moins accentuée des oreilles, de la dyspnée et de la polypnée, ete. ${ }^{11}$ ).

Walter Edmunds hat auch meine Experimente wiederholt und gefunden dass: „if the thyroid and parathyroids are removed the animals as a rule die: if the parathyroids are alone removed no obvious result fellows: if the thyroid is removed and the parathyroids left some of the rabbits live and some die: some of these latter die with symptoms of chronic myxoedema, the hairs falls out and a remarkable swelling occurs in the lower part of the face". Andererseits sagt auch Edmunds (Journ. of pathol. aud bacteriol,, janvier 1896): "These experiments of Gley's have been repeated by myself, and it was found that if both the thyroid and the parathyroid glands are removed the animals die; out of a batch of 7 rabbits, on whom this operation was performed, 5 died within 8 days, the other 2 surviving for months."

Endlich hat $\mathrm{C}$ a p o b i a n c o einige convulsive Phänomenen beobachtet: "questi animali (Kaninchen) soccombeno non meno dei cani alla tiroidectomia totale. Il numero grande delle tiroidi soprannumerarie, la grande varietà della sede spiegano le erronee interpretazioni per lunga pezza prevalse.

1) Die detaillirte Arbeit Rouxeau's erscheint in der nächsten Num. mer, 1. Januar 1897, der "Arehives de Physiologie" unter dem Titel: Relation de 103 opérations de thyroidectomie chez le lapin. 
Bemerkungen über die Funktion der Schilddrüse u. ihrer Nebendrüsen. 315

„Al G ley spetta il merito di aver per primo proclamata la niuna refrattarietà di conigli all' ablazione delle tiroidi. I suoi lavori comparvero quando io già aveva in corso indagini, che mi avevano convinto contro la pretesa immunità.

„Prescindendo dalla diminuzione del peso del corpo, che in taluni casi è rilevante, in questi roditori i disturbi si accentrano nella sfera respiratoria. Sovra 30 conigli, operati nell' Istituto diretto dal prof. $\mathrm{Pa}$ a $\mathrm{d}$ in 0 , non mi è riuscito sorprendere in niun caso un solo accesso convulsivo. Contrazioni fibrillari, limitati spasmi lievissimi, ma null'altro che potesse richiamare a mente lo stato dei cani in analoghe condizioni. I disturbi respiratori sono, invece, dei più caratteristici.“.

Sogar Mo a s $\mathrm{u}^{1}$ ), der bekanntlich früher meine Ansichten bekämpft hat, hat selbst tetanische Störungen bei 2 Kaninchen auf 1 Dutzend operirter beobachtet.

Diese zahlreichen Bestätigungen meiner Experimente genïgen gewiss zum Beweise datür, dass, wenn die Versuchsthiere der Herren B. und J. sich nicht wie die Pariser Thiere verhalten, so doch diese letzteren nicht viel von denen in Halle, Bern, Gent, Lyon, Nantes, London, Napoli differiren.

B. und J. sagen, dass, wenn ich meine Untersuchungen vermehren wirde, meine Statistik immer mehr der ihrigen gleichen wïrde. Das ist mehr als zweifelhaft, da meiner Statistik diejenige Rouxeau's. (die sich über 103 Operationen erstreckt) gleicht, so dass ich meinerseits mit mehr Recht behaupten kann, dass die Resultate der Herren B. und J. im Gegentheil den meinen gleichen würden, wenn sie ihre Versuche über die Glandula vermehren wiirden. Deshalb verstehe ich nicht, warum sie sagen: „Gle y vermeidet es ubrigens durchaus, eine Erklärung für den ander. weitigen Ausfall seiner späteren Operationen zu geben." Ich antworte darauf, dass ich die Erklärung nicht vermieden, sondern dass ich keine gegeben habe, weil gegenwärtig kein Physiolog eine Erklärung davon abgeben kann, warum gewisse Thiere, seien es Hunde oder Kaninchen, je nach noch nubekannten Umständen, die Thyreoidectomie iberleben.

1) G. Moussu: Effets de la thyrö̈dectomie chez nos animax domestiques (Mémoires de la Société de Biologie, 1892, p. 271). 
2. Der zweite Punkt betrifft die Nebenschilddriisen. Eine vor meinen Versuchen gut bewiesene Thatsache ist die der Unschädlichkeit der Thyreoidectomie beim Kaninchen. Sicherlich haben unsere Autoren Todesfälle bei einfacher Thyreoidectomie beobachtet. Ich habe selbst solche Erfahrungen gemacht; Rouxeau und W. E d m unds auch; aber in diesen Fällen trat in der Regel eine chronische Krankheit, nämlich typische Cachexie, ein. Bei totaler Thyreoidectomie tritt im Gegentheil eine acute Krankheit convulsiver Natur auf.

Diese letztere Thatsache wird nun eben von den Verfassern bestritten. Was ist nun vorgefallen in den vier einzigen Fällen, in denen sie die Nebendriisen bestehen liessen? Sie erkennen selbst an, dass sie bei der Section gefunden haben, dass die Glandulae sich nicht entwickelt hatten. Sie sagen, dieselben seien "normal" oder "klein und blass" gewesen. Ich habe auch Fälle beobachtet, wo die Glandulae sich nicht entwickelt haben. Es kommt übrigens vor, dass diese Organe mit der Thyreoidealappen derselben Seite gemeinsame Gefässe haben, wenn sie ganz nahe an den Lappen lagen. In solchen Fällen ist es ziemlich schwer, diese Gefässe nicht zu unterbinden, sodass dadurch die Funktionen der Glandulae nnterbrochen werden, was einer totalen Thyreoidectomie gleichkoumt; und selbst in solchen Fällen können einzelne Thiere überleben. Ich habe ein Kaninchen beobachtet, das 4 Monate nach der Operation gelebt hat. Bei der Section habe ich normale, nicht hypertrophirte Glandulae gefunden. Aber es giebt auch einige Hunde, welche der Thyreoidectomie widerstehen, und bei welchen man bei der Section keine accessorischen Drüsen findet. Diese bekanntlich seltenen Fälle beweisen übrigens nur, dass die Unterschiede im Metabolismus mitunter je nach den Individuen beträchtlich sind. Warum doch die Fraige der Verfasser: "Wenn so viele Thiere (12 von 55) trotz Entfernung der Nebendrisen nicht ihr Leben verlieren, wo bleibt dann die für das Leben entscheidende Stellung der Nebendrüsen?" (S. 35). Denn man könnte mit den alten Autoren zugeben, dass die Stellung der Schilddrüsen, weil einige Hunde trotz Entfernung dieser Organe nicht ihr Leben verlieren, nicht für das Leben entscheidend ist.

Eine weniger wichtige Frage ist es, zu wissen, ob eine einzige Nebendriise geniigt, die Thiere von den Folgen der Thyreoidectomie zu behiiten. B. und J. behaupten, dass dies nicht der Fall 
Bemerkungen über die Funktion der Schiiddrüse u. ihrer Nebendrüsen. 317

ist, und schliessen daraus dass diese Organe zur Erhaltung des Lebens unnöthig sind. Aber ich habe schon selbst gesagt, dass nur ein einziges dieser Organe nicht immer genügt. Hier ist ein Beispiel:

Versuch.

29. März 1893. 2 Kaninchen mittleren Alters. Thyroïdectomie ohne Narkose. Die Glandula der rechten Seite bleibt bei beiden erhalten.

3i. März, Morgens, wurde das eine todtgefunden.

Das andere durch den.April abgemagert und von Parese befallen. Den 16. Mai, Dyspnoë; es ist vollständig lahm und stirbt Abends, - Die Lungen sind normal. Gewicht der Glandula $=0,005 \mathrm{gr}$.

Hofmeist e r, der dies auch beobachtet hat, anerkennt, dass ich gesagt habe: „Demnach ist dieser Fall vielleicht zu denjenigen zu rechnen, auf welche Gley neuerdings aufmerksam gemacht hat, dureh die Angabe, dass eine Nebendrise nicht immer genïge, um das Thier zu retten." Im übrigen handelt es sich hier um allgemeine Thatsachen. Wenn man Thieren die Leber exstirpirt, darf man nicht mehr als ein gewisses Volumen ausschneiden, und aus der Thatsache, dass das iuberbleibende Stiick nicht zur Erbaltung des Lebens geniigt, schliesst man doch anch nicht, dass die Leber nicht ein zur Lebenserbaltung nothwendiges Organ sei. Und ist es nicht dasselbe mit der Niere, wo man doch schon gesehen hat, dass Thiere sterben, selbst wenn man ihnen eine halbe oder gar zuweilen eine ganze Niere gelassen bat?

Andererseits baben vier Fälle den Autoren zu der Erklärnng genuigt, dass durch Thyreoidectomie in den Nebenschilddrisen keine Veränderungen hervorgerufen werden. Nun baben seit meinen Versuchen Verstraeten und Vanderlinden, Walter E d m u nds, Ro ux e a gleichfalls Modificationen constatirt; und Mo u s s u selbst erkennt an, dass diese Organe sich congestionniren. Die Hauptfrage, um die es sich gegenwärtig handelt, ist die Frage nach den Zellveränderungen in den Nebenschilddrïsen. Das glaubte ich zuerst nach einigen histologischen Untersuchungen, zu einer Zeit, wo die embryonare Natur dieser Organe unzweifelhaft schien. In der That scheinen sie zusammengesetzt zu sein ans einer homogenen Masse gleichförmiger epithelialer Zellen. Da nun nach der einfachen Thyreoidectomie die Gefässe und die Bindegewebe im allgemeinen in jener Masse sich vermehren, so theilt sich die Masse in Zellenstränge, und gewisse Zellen scheinen gleichzeitig umfang- 
reicher. Diese Veränderungen, wie ich sie hier kutr zusanmenfasse, sind wahrscheinlich ungeniigend, um zu beweisen, dass die Glandula den Typus der Drüse zu erreichen strebt. Wenigstens bin ich später zu dieser Schlussfolgerung mit meinem Mitarbeiter, Professor Nicolas, gekommen. Es muss übrigens gesagt werden, dass der wirkliche Ursprung dieser Nebenschilddriisen auch heute noch nicht ganz klar ist. Sicher ist, dass die Diskussion ein Ende nehmen wiirde, wenn die Embryologen uns darüber sicheren Aufschluss geben könnten.

Nun möchte ich bemerken, dass die Autoren sich anscheinend mehrmals bestreben, meine frühere Arbeit mit meinen späteren Mittheilnngen in Widerspruch zu bringen. Das ist rein polemisch und folglich ohne Interesse. Ich will nur darauf aufmerksam machen, dass ich, wie alle Untersucher, immer nach meinen jeweiligen Versuchen geschlossen habe. Das habe ich anch bei meiner ersten Arbeit gethan, wo ron 16 Kaninchen nur eines überlebte. Später, als ich von einer ersten Serie von 16 und einer zweiten von 23 mehrere Thiere überleben sah, habe ich das einfach konstatirt. Ich sehe nicht ein, wie man sich dadurch, dass man Thatsachen konstatirt, in Widersprüche verwickeln könnte. Es giebt vielleicht Experimentatoren, die mit den 16 ersten Versuchen befriedigt gewesen wären; ich habe im Gegentheil meine Versuche weitergefihht; das ist der Grund, weshalb sich meine Resultate geändert haben, und sie würden sich vielleicht nochmals ändern, wenn das Experiment es verlangte.

Addendum. - Im völligen Widerspruch mit dem, was B l u mreich und Jacoby behaupten, scheinen jetzt die Glandulae parathyreoideae noch wichtiger, als ich glaubte. Schon 1893 hatte ich gezeigt, dass die Erhaltung der Glandulae externae bei Hunden genüge, dieselben vor den Folgen der Thyreoidectomie zu behïten ${ }^{1}$. Vassale und Generali haben kürzlich bewiesen, dass nach Exstirpation der vier Glandulae (S a ndström-Gley's Glandulae und Kohn's innere Körperchen) bei Hunden und Katzen diese Thiere sterben ${ }^{2}$ ). Ich habe dieses Resultat kontrol-

1) E. Gley: Recherches sur le rôle des Glandules thyroïdes chez le chien (Arch. de physiol., 5. série, V, p. 766; 1893).

2) G. Vassale und F. Generali: Sugli effetti dell' estirpazione delle ghiandole paratiroidee (Riv. di patol. nerv. e men- 
Bemerkungen über die Funktion der Schilddrüse u. ihrer Nebendrüsen. 319

lirt $\left.{ }^{1}\right)$; Mouss u hat seinerseits dasselbe konstatirt ${ }^{2}$ ). Endlich haben wir beide, Roux e a und ich, unabhängig von einander gezeigt, dass die Ausschneidung der Glandulae beim Kaninchen letalen Ausgang herbeiführen kann ${ }^{3}$ ). Ich habe auch eine Erklärung dafür vorgeschlagen, warum diese Operation in der That nicht immer bei diesem Thiere den Tod herbeiführen muss ${ }^{4}$ ).

Die Frage, um deren Stellung, wie ich gesagt habe ${ }^{5}$ ), es sich handelt, ist also folgende: ob die ganze thyroidale Function nicht den Glandulae zuzuschreiben ist, oder ob diese kleinen Organe nicht mit der Schilddrise eine gemeinsame Function zu vollziehen haben, oder schliesslich ob wir nicht, wie Moussu glaubt ${ }^{6}$ ), mit zwei verschiedenen, specifischen Verrichtungen, mit einer thyroidalen und einer parathyroidalen, zu thun haben.

tale, 1, p. 95, marzo 1896 und p. 249, loglio 1896; und Arch. ital. de Biol., XXV, p. 459 und XXVI, p. 61; 1896.

.1) Soc. de Biol., 9 und 16 janvier 1897, p. 18 und 46.

2) Soc. de Biol., 16 janvier 1897, p. 44.

3). Rouxeau, Soc. de Biol., 9 janvier 1897, p. 17; Gley, Ibidem, p. 18 .

4) Soc. de Biol., 9 janvier 1897.

5) Loc. cit.

6) Soc. de Biol., 16 und 23 janvier 1897. 